University of South Florida

DIGITAL COMMONS

@ UNIVERSITY OF SOUTH FLORIDA
Digital Commons @ University of South Florida

\title{
Florida Transit Handbook 2002
}

CUTR

Follow this and additional works at: https://digitalcommons.usf.edu/cutr_nctr

\section{Scholar Commons Citation}

CUTR, "Florida Transit Handbook 2002" (2002). Research Reports. 188.

https://digitalcommons.usf.edu/cutr_nctr/188

This Technical Report is brought to you for free and open access by the National Center for Transit Research (NCTR) Archive (2000-2020) at Digital Commons @ University of South Florida. It has been accepted for inclusion in Research Reports by an authorized administrator of Digital Commons @ University of South Florida. For more information, please contact digitalcommons@usf.edu. 


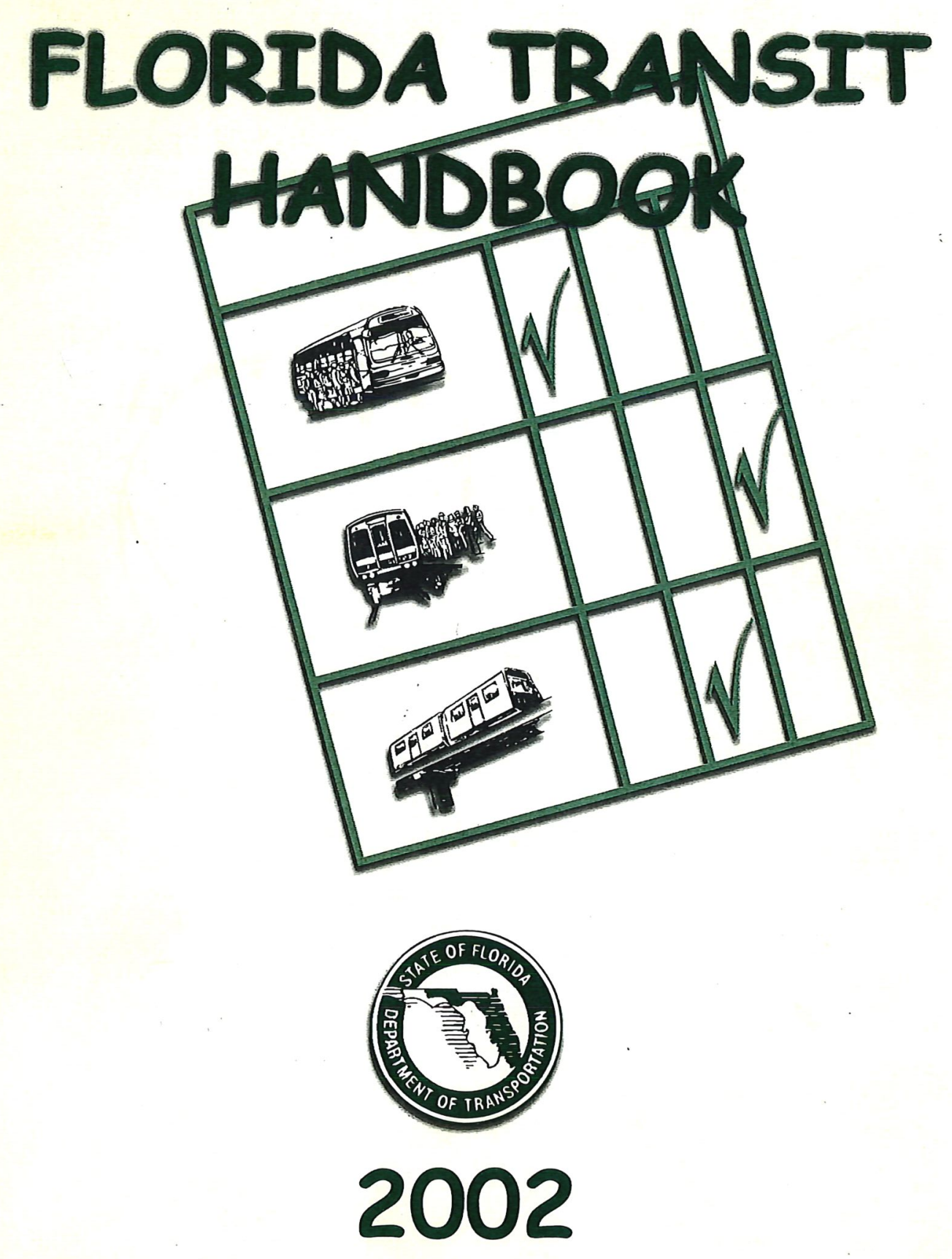




\section{PUBLIC TRANSIT IN FLORIDA}

his Handbook offers a general overview of public transit in the State of Florida. The information in the following pages provides insight into transit's contribution to meeting the statewide transportation objectives and mission of the Florida Department of Transportation (FDOT).

Public transit plays an important role in maintaining the livability of Florida's growing communities. Transit not only helps alleviate traffic congestion and improve air quality, but also provides mobility for many of Florida's citizens, as well as visitors and tourists to the Sunshine State.

By providing mobility for many Floridians, transit also plays a large part in helping former welfare recipients and others gain better access to employment and school-starting them on the road to self-sufficiency. Together, Florida's 26 transit systems provided transportation for more than 190 million trips during fiscal year 2000. This handbook includes 23 transit agencies since it focuses on the fixed-route services of Florida's urban transit systems. Although 20 of these 23 fixed-route systems also provided demand-response services, the following data represent only the fixed-route portion.

For more information on FDOT and public transit in Florida, please visit the FDOT website: http://www11.myflorida.com, or call (850) 4I4-4500.

\section{FDOT Mission}

Our mission is to provide a safe transportation system that ensures the mobility of people and goods, enhances economic prosperity and preserves the quality of our environment and communities. 


\section{FLORIDA TRANSIT \\ HANDBOOK}

\section{Contents}

FDOT Public Transit Office 3

FDOT District Public Transportation Offices

Florida's Transit Systems

Transit System Profiles

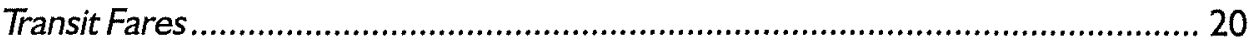

Transit System Summary .............................................................................. 2 I

Glossary of Terms

\section{FDOT Public Transit Office}

The FDOT Transit Office consists of three sections (Transit Planning; Grants Administration; Transit Operations), each of which has specific areas of responsibility.

\section{Transit Planning}

The Transit Planning unit is responsible for statewide transit planning, policy analysis, performance monitoring, plan review, technical assistance, research, and interagency planning coordination.

\section{Grants Administration and Commuter Assistance}

The Grants Administration and Commuter Assistance unit provides financial and technical assistance to Florida's transit agencies and commuter assistance services. Staff also coordinates with and provides technical assistance to the Commission for the Transportation Disadvantaged. This unit's responsibilities fall into eight areas: Federal Transit Administration coordination; grant management; financial management; rural transit technical assistance; urban transit technical assistance; innovative finance; commuter assistance; and legislative analysis.

\section{Transit Operations}

The Transit Operations unit is responsible for developing and implementing transit programs that provide services and technical assistance to support transit operational and maintenance functions and activities. This unit also develops standards and oversees compliance with State and Federal regulations.

Additional transit information can be found at the following web addresses: FDOTWebpage

http://www11.myflorida.com/transit

Transit 2020: Florida's Strategic Plan for Public Transportation http://www11.myflorida.com/transit/Pages/transit2020plan.htm

Florida Transit Forum

http://www11.myflorida.com/transit/Pages/floridatransitforum.htm 
Florida Vehicle Procurement Program (FVPP)—The FVPP provides Florida's public transportation providers with the opportunity to purchase vehicles as a consortium. For more information, please visit: http://cutr.usf.edu/research/fvpp.htm

Florida Maintenance Training Program http://cutr.usf.edu/research/fmtp/fmtp.htm

State Bus Fleet Program-The purpose of this program is to establish the requirements for the lease and management of State Public Transportation Fleet Equipment. For more information, please visit: http://www11.myflorida.com/transit/Pages/ statebusfleetprogram.htm

Substance Abuse Review

http://www11.myflorida.com/transit/Pages/SubstanceAbuseReview.doc

Transit Safety Programs

http://www11.myflorida.com/transit/Pages/transitsafetyprograms.htm

Commuter Assistance Program

http://www.commuterservices.com

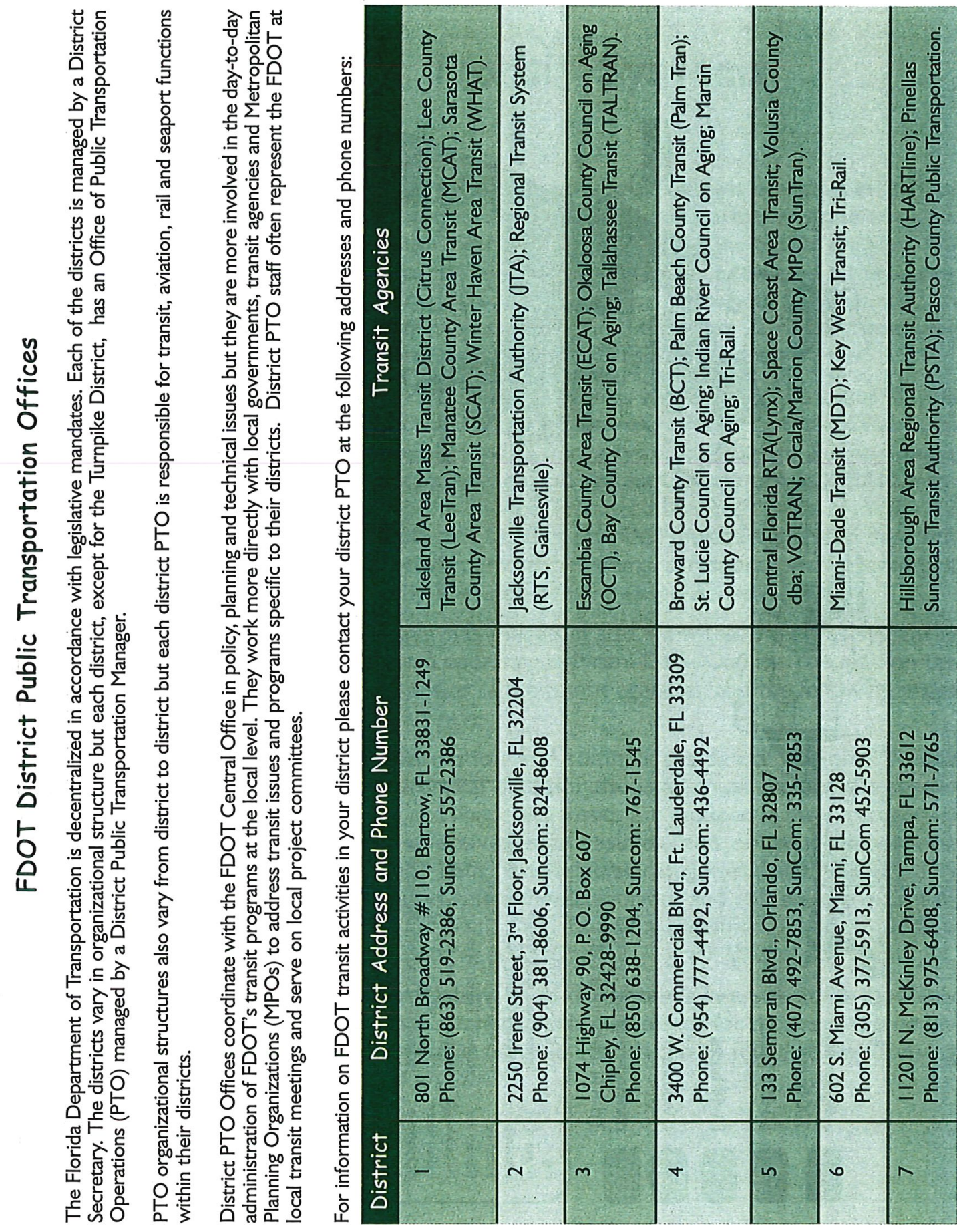




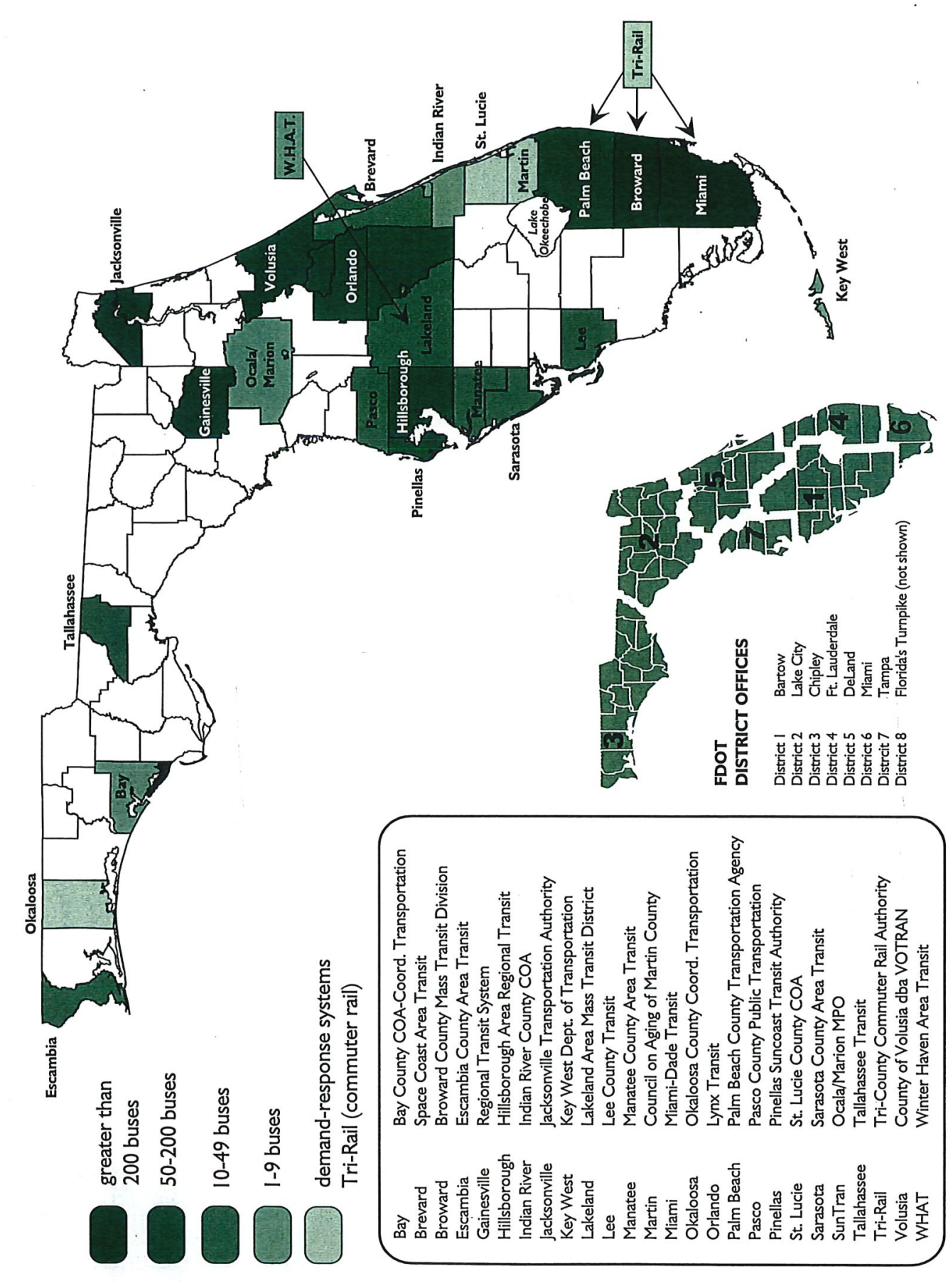

\section{Florida's Transit Systems}

n fiscal year (FY) 2000, there were 23 fixed-route transit systems operating in Florida.

One of these systems, Tri-County Commuter Rail Authority,provides regiona

rail service between Palm Beach, Broward, and Miami-Dade Counties In addition, Miami-Dade Transit operates a heavy rail system (Metrorail) and an automated guideway, or peoplemover, system (Metromover). Jacksonville Transportation Authority operates an automated guideway system (Skyway Express), as well. Of the other 22 systems operating fixed-route service, 20 also offer some type of demand-response service.

To meet their service objectives, transit agencies across the United States and Florida sometimes find it necessary to contract the operation of portions of their services to private operators. In FY 2000, three of Florida's transit systems, Broward County Transit and Palm Beach County Transportation Agency, and Volusia COunty dba VOTRAN, contracted for regular fixed-route motorbus service. Privatized service is more common for demand-response service. In FY 2000, nine systems provided privately-operated demandresponse service, three systems directly-operated their demand-response service, and eight provided both purchased and directly-operated demand-response service.

During FY 2000, Florida's transit agencies ranged in size from the three-bus system in Panama City to the 625 vehicle system operating in Miami-Dade County. The map on the previous page shows the location and size of the transit agencies, and the following pages contain brief profiles of each of the agencies.

Each profile provides contact information and summarizes select National Transit Database (NTD) information. System total data (for the fixed-route modes) are shown except where noted. The operating and financial statistics are grouped in three categories: general performance indicators, effectiveness measures, and efficiency measures. General performance indicators report data in the selected categories that are required by NTD reporting. Effectiveness measures refine the data further and indicate the extent to which various service-related goals are being met. Efficiency measures provide an indication of the level of resources (labor, cost) needed to achieve a given level of service output.

Following the profiles is a page summarizing all of the system's fare structures as of July 2002. After the fare information, data summarizing transit trends for the state as a whole are presented. More information on public transit in Florida can be found at the following website: http://www11.myflorida.com/transit. 
Bay County Council on Aging, Inc. (Bay Town Trolley)

Ms. Elizabeth Coulliette, Executive Director

(850) 769-3468 wnw. baytowntrolley.com

Bay County Council on Aging, Inc., operates the Bay Town Trolley, which serves Bay County with fixed-route motorbus services. In addition, demand-response services are provided (Bay Coordinated Transportation).

\section{General Performance Indicators (FY 2000)} Service Area Population

Service Area Population Density

Annual Passenger Trip

Annual Revenue Miles

Effectiveness Measures (FY 2000) assenger Trips Per Capit

Passenger Trips Per Revenue Mile

Efficiency Measures (FY 2000)

Operating Expanse Per Capit

Operating Expense Per Trip

Operating Expense Per Rev. Mile
$1,565.6$

58,158

Route Miles

Peak Vehicles

Operating Expens

perating Revenue

3

$\$ 225,434$

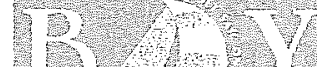

0.54 Rev Miles Between Safety Incidents (zero incidents reported)

0.42 Rev. Miles Between Failures

11,297

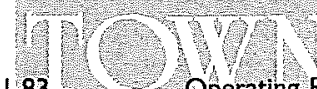

Operating Revenue Per Operating Expens

$\$ 3.39$ Passenger Trips Per Employee FTE

Average Fare
Escambia County Area Transit (ECAT)

Ms. Nedra Woodyatt, Resident Manager

(850) 595 Firfield Drive, Pensacola, FL 3250

Escambia County Area Transit is a department of the county government. The department is governed by a five-member elected Board of County Commissioners. In addition to providing fixed-route motorbus service, ECAT contracts to provide demand-response transportation services.

Service Area Population Indicators (FY 2000)

261,647

Al,633:3

Annual Passenger Thips

Operating Expense

Operating Eevenue

(387.13$$
\text { Son. }
$$

Effectiveness Measures (FY 2000)

assenger Trips Per Capita

6.23

Rev Miles Between Safety Incidents

venue Mile

106,724

Efficiency Measures (Fr 2000)

Operating Expense Per Capita

Operating Expense Per Rev. Mile

\section{Broward County Mass Transit Division (BCT) Mr. Robert Roth, Director \\ 3201 West Copans Road, Pompano Beach, FL 3306 \\ (954) 357-8361 uww.broward.org/bct}

Broward County Mass Transit Division is part of the Broward County Community Services Department. It provides fixedroute motorbus service throughout Broward County. Connections with Palm Tran (in Palm Beach County), Miami-Dade

Transit, and Tri-Rail (commuter rail service) are also provided. 'In addition, a contracted paratransit service is offered.

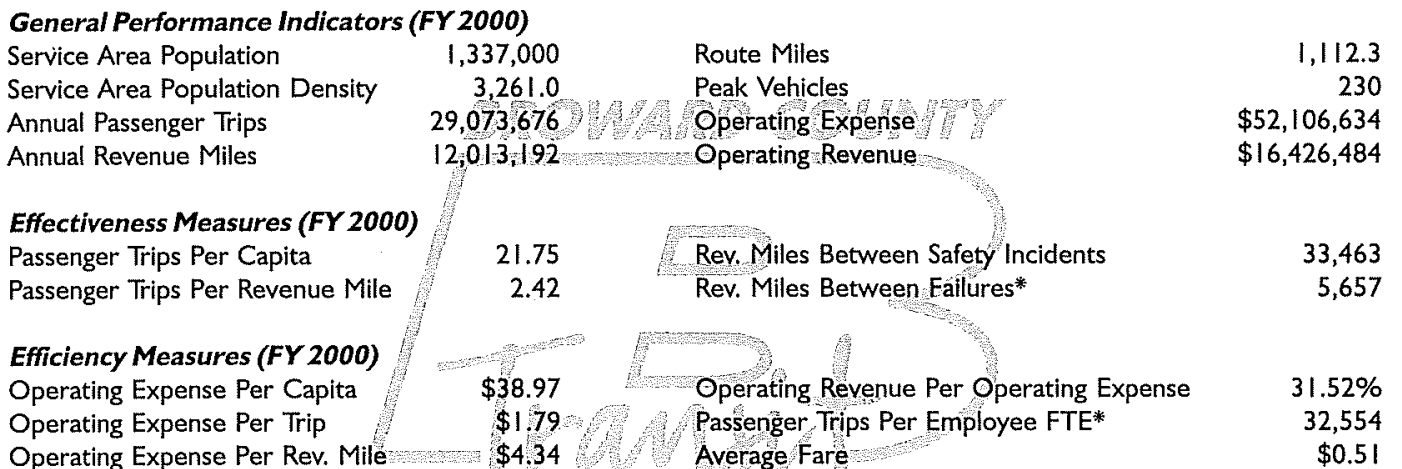

(6),634

\section{Hillsborough Area Regional Transit Authority (HARTline) Ms. Sharon Dent, Executive Director}

(813)623-5835 munhartline org

Hillsborough Area Regional Transit is an independent authority that is governed by an eleven-member Board of Directors. The authority serves the transit needs for Hillsborough County excluding Plant City. HART provides fixed-route motorbus service and paratransit service.

General Performance Indicators (FY 2000) Service Area Population $\quad 1,001,910$

Annual Revenue Miles $\quad 5,824,416$

Route Miles

eak Vehicles

Operating Expense

Operating Revenue

879.2

Effectiveness Measures (FY 2000)

Passenger Trips Per Capit

2

Passenger Trips Per Reyenue Mile

Rev. Miles Between Safety Incidents $\quad 34,877$

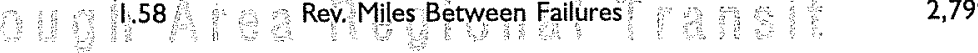

Operating Expense Per Capit

Operating Expense Per Rev. Mile

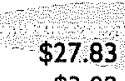

$\$ 3.02$

$\$ 3.02$

Operating Revenue Per Operating Expense $\quad 24.57 \%$ $\begin{array}{lr}\text { Passenger Trips Per Employee FTE } & 19,100\end{array}$ Average Fare
Service Area Population Density $\quad 3,670.0$

Annual Passenger Trips $\quad 9,219,738$ 
Indian River County Council on Aging, Inc. (Indian River Transit/Community Coach) Mr. Robert Jacobson, Transportation Supervisor

(56I) 569-0760 www.indian-river.fl.us/coa/transportation.htm

Indian River County Council on Aging, Inc., operates in a quasi-government status under the five-member elected Board of County Commissioners as the Transit Element of Indian River County. It is governed by a volunteer Board of Directors. Community Coach serves the rural and urbanized portions of Indian River County. The system provides point deviation hours, and weekend demand-response services are contracted stretch

\section{General Performance Indicators (FY 2000)}

Service Area Population

Service Area Population Density

Annual Passenger Trips

Annual Revenue Miles

Effectiveness Measures (FY 2000)

assenger Trips Per Capita

Passenger Trips Per Revenue Mile

Efficiency Measures (FY 2000)

Operating Expense Per Capit

Operating Expense Per Rev. Mile
(0,000

\begin{tabular}{l|l}
153,768 & Operating Expense \\
226,524 & Operating Revenue
\end{tabular}

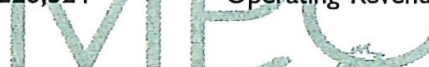

1.41 Rev. Miles Between Safety Incidents

$0.68 \quad$ Rev. Milés Between-Failures Rev. Miles Between-

$\$ 5.03 \square$ Operating Revenue Per Operating Expens $\$ 3.57$ - Passenger Tríps Per Employee FTE

$\$ 2.42 \quad$ Average Fare
Key West Department of Transportation (KWDOT)
Ms. Myra Hernandez, Director Ms. Myra Hernandez, Director

(305) 292-8247 wuw.keywestcity.com/depts/dot/dot.htm/

Key West Department of Transportation is a department of the City of Key West. The department is managed by the reports to the seven-member City Commission. The transit system provides fixed-route motorbus service to the City o Key West and to Stock Island. Demand-reponse service is available throughout Monroe County. Shuttle service from a park-and-ride facility to the downtown historic areas of Key West is provided for tourists.

General Performance Indicators (FY 2000)

Service Area Population

Service Area Population Density

Annual Passenger Tips

Effectiveness Measures (FY 2000)

Passenger Trips Per Capita

Passenger Trips Per Capita

Efficiency Measures (FY 2000)

Operating Expense Per Capita

Operating Expense Per Trip

Operating Expense Per Rev. Mile

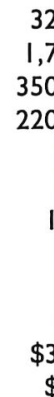

350,222 Operating Expense

,548 la Gucoperating Reve

Cayo Hiveso

$10.79 \quad$ Rev. Miles Between Safety Incidents

Rev. Miles Between Failures

$\$ 37.35 \quad$ Operating Revenue Per Operating Expense $\quad 9.27 \%$

$\$ 3.46$ Passenger Trips Per Employee FTE (employees not reported)

$\$ 5.50$ Average Fare
Jacksonville Transportation Authority (JTA)

Mr. Derek Morse, Executive Director

100 N. Myrtle Avenue - P.O. Drawer "O", Jacksonville, FL 32203

(904) 630-3181 www.jtaonthemove.com

Jacksonville Transportation Authority is an independent authority that is governed by a seven-member, appointed Board of transportation services include fixed-route motorbus, automated guideway (Skyway Express), and demand-response.

\section{General Performance Indicators (FY 2000)}

834,337

$3,447.7$

$7,455,869$

Effectiveness Measures (FY 2000)

Passenger Trips Per Cap

Passenger Trips Per Revenue Mile

Efficiency Measures (FY 2000)

Operating Expense Per Cap

Operating Expense Per Thip
Service Area Population Density
Route Miles

Peak Vehicles

, 103.7

$\$ 32,273,506$

$\$ 6,777,298$

33,137
5,273

Rev. Miles Between Failures

$\$ 38.68$ Operating Revenue Per Operating Expense

$\$ 3.69$

$21.00 \%$ assenger Trips Per Employee FT

\section{Lakeland Area Mass Transit: District (Citrus Connection) \\ Mr. Steven Githens, Transit Director}

(863) 688-7433 www.maps.polk.net/transit/transport.htm/

Lakeland Area Mass Transit District is an independent authority. The authority is governed by a five-member Board of (1) and vanpool services.

General Performance Indicators (FY 2000)

Service Area Population

Service Area Population Density $\quad 1,428,6$

Annual Passenger Trips $\quad 1,358,985$

Annual Revenue Miles

$1,099,45$

Route Miles

Peak Vehicles

Operating Revenue

Passenger Trips Per Revenue Mile
Effectiveness Measures (FY 2000)

Passenger Trips Per Capita

Efficiency Measures (FY 2000)

Operating Expense Per Capita

Operating Expense Per Rev. Mile

$-1$ 1.24

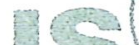

Rev. Miles Between Safety Incidents (zero incidents reported) Rev. Miles Between Failures 5,756
2.03

$\$ 2.5$ \begin{tabular}{lr} 
Operating Revenue Per Operating Expense & $26.56 \%$ \\
Passenger Trips Per Employee FTE & 20,238 \\
\hline
\end{tabular} 
Lee County Transit (LeeTran)

0715 East Airport Road, Fort Myers, FL 33907

(941) 277-5012 www.leegov.com

Lee County Transit is an independent division of the Lee County Government. The division is governed by the Lee County Board of County Commissioners, a five-member public policy body. LeeTran provides public transportation services to Lee County via fixed-route motorbus services and handicapped transportation services. In addition, LeeTran contractually provides for seasonal fixed-route motorbus service.
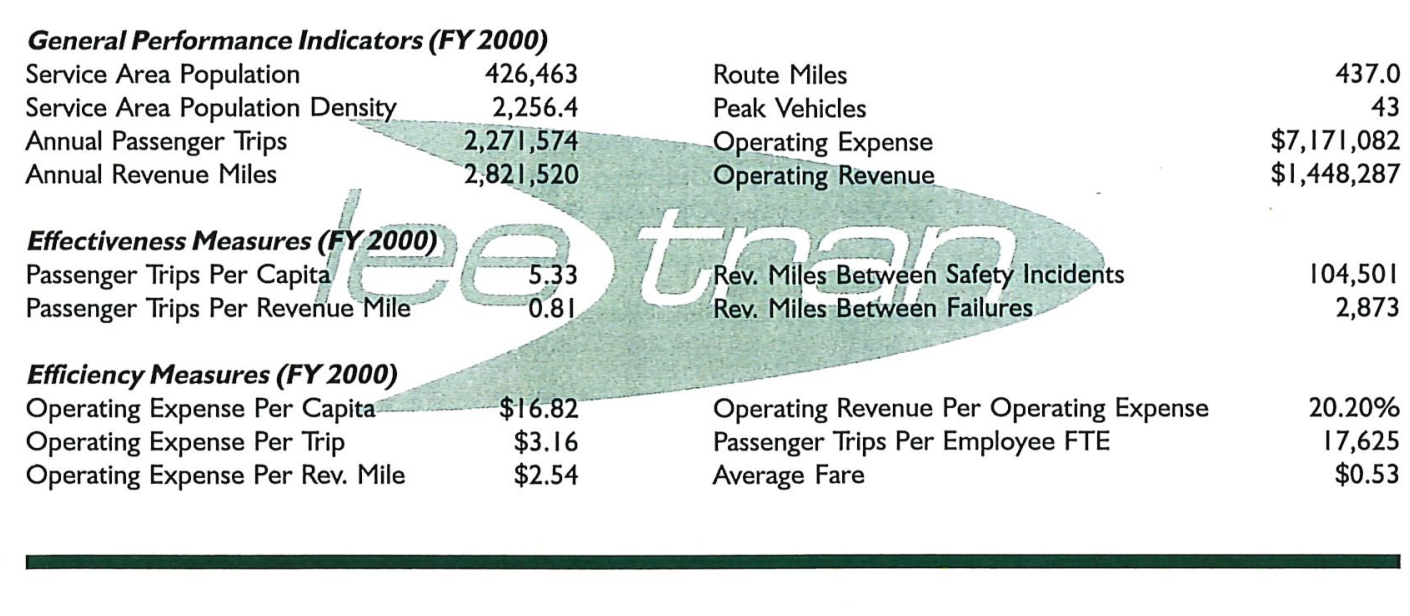

\section{Lynx Transit
Mr. Byron Brooks, Executive Director}

445 West Amelia Street, Suite 800, Orlando, FL 32801

(407) 841 -2279 unw.golynx.com

LYNX is an independent authority that is governed by a Board of Directors. The authority provides service to Orange County (including Orlando. Winter Park, Maitland, and others), Seminole County (including Altamonte Springs, Casselbern and Sanford), and Osceola County (including Kissimmee and St. Cloud). LYNX provides fixed-route motorbus service, paratransit service, transportation-disadvantaged coordination, a five-county regional ridesharing assistance program, and vanpool service.

\section{General Performance Indicators (FY 2000)}

Service Area Population Density

Annual Passenger Trips

$1,357,852$
535.0
$20,735,614$

$20,735,614$

Annual Revenue Miles

Effectiveness Measures (FY 2000) Passenger Trips Per Capita Passenger Trips Per Revenue Mile

$0,967,378$
Route Miles Peak Vehicles

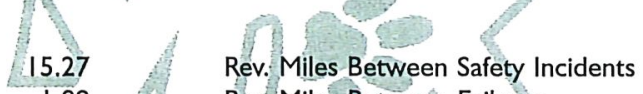

5.27 Rev. Miles Between Safety Incide

viles Between Failure

Efficiency Measures (FY 2000)

Operating Expense Per Capita

Operating Expense Per Trip

Operating Expense Per Rev. Mi
Manatee County Area Transit (MCAT)

Mr. Pest Gajds, Tansil Section Manager

(941) 747-8621 mwwcomanateeflus/tas

Manatee County Area Transit is a division within the Community Services Department of Manatee County. The division is governed by the Board of County Commissioners. MCAT provides service to the urbanized parts of Manatee County vit fixed-route motorbus services and demand-response services.

General Performance Indicators (FY 2000)

$\begin{array}{lll}\text { Service Area Population } & \text { 253,207 } & \text { Route Miles }\end{array}$

$\begin{array}{lrr}\text { Service Area Population Density } & 339,0 \\ \text { Annual Passenger Trips } & 655,984 & \begin{array}{l}\text { Peak Vehicles } \\ \text { Ond }\end{array} \quad \text { Operating Expe }\end{array}$

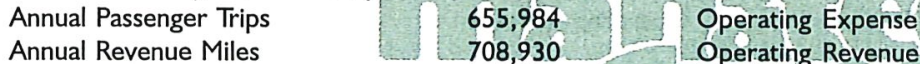

166,8
12

(6)

Effectiveness Measures (FY 2000)

Capita 125

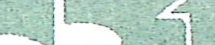

Rev. Miles Between Safety Incidents

Rev. Miles Between Safety $/$ nile
Rev. Miles Between Failures

$\$ 2,326,877$

Passenger Trips Per Revenue MI

Efficiency Measures (FY 2000)

Operating Expense Per Capita : $\$$. $\$ 9.19$.

Operating Expense Per Trip $\cdots 33.5$

Operating Expense Per Rev. M

$\$ 3.28$

Operating Revenue Per Operating Expense

Passenger - Trips Per Employee FTE

Average Fare
236,310

8

$8.80 \%$

$\$ 0.42$

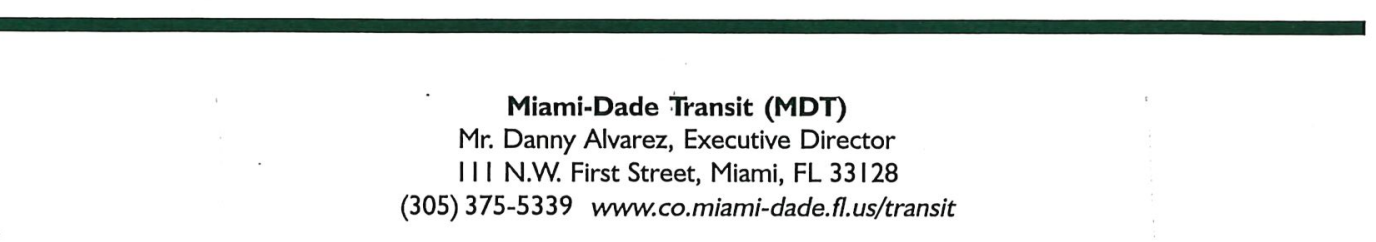

Miami-Dade Transit operates within Miami-Dade County as well as three routes which extend into Broward County. The agency is governed by Miami-Dade County. MDT provides countywide services throughout Miami-Dade County and municipal services to those who live in the unincorporated area of the county. It is responsible for the management, construction, and operation of Metrorail, Metrobus, Metromover, and Paratransit Operations.

$\begin{array}{lrlr}\text { General Performance Indicators } & (\text { FY 2000) } & & \\ \text { Service Area Population } & 1,800,000 & \text { Route Miles } & 1,705.9 \\ \text { Service Area Population Density } & 6,315.8 & \text { Peak Vehicles } & 625 \\ \text { Annual Passenger Trips } & 84,131,473 & \text { Operating Expense } & \$ 216,792,635 \\ \text { Annual Revenue Miles } & 31,187,342 & \text { Operating Revenue } & \$ 73,708,834\end{array}$

Annual Revenue Miles $\quad 31,187,342=$ Operating Revenue

Effectiveness Measures (FY 2000)

Passenger Trips Per Capita $\quad 46.74 \quad$ Rev. Miles Between Safety Incidents $\quad 39,933$

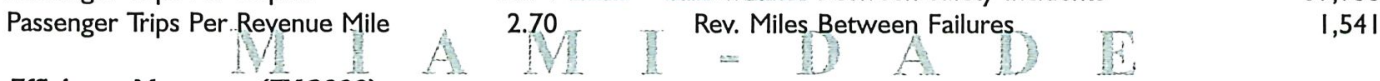

\begin{tabular}{l|llr} 
Efficiency Measures (FY 2000) & & & \\
Operating Expense Per Capita & $\$ 120.44$ & Operating Revenue Per Operating Expense & $34.00 \%$ \\
Operating Expense Per Trip & $\$ 2.58$ & Passenger Trips Per Employee FTE & 33,469
\end{tabular}

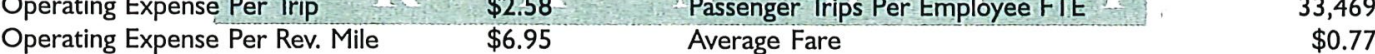


Ocala/Marion County Metropolitan Planning Organization (SunTran) Mr. Greg Slay, Director

(352) 629-8529 mww.suntran.org

SunTran is a newer transit system that started operating in December 1998. The system is governed by the Ocala/Marion County MPO and provides purchased fixed-route motorbus service on five routes in Ocala and Silver $S$ prings Shores.

\section{Senvice Area Population \\ Service Area Population Density \\ Annual Revenue Miles}

Effectiveness Measures (FY 2000) Passenger Trips Per Capita

Efficiency Measures (FY 2000)

Operating Expense Per Capita

ating Expense Per Trip

Operating Expense Per Rev Mile
$1,273.4$

154,719
317,548

2.61

0.49

$\$ 11.93$

$\$ 2.22$
Route Miles

Peak Vehicles

Operating Revenue

108.2

$\$ 706,303$

$\$ 134,20$

Miles Between Safety lncidents:

158,774

Operating Revenue Per Operating Expense $\quad 19.00 \%$ Passenger Trips Per Employee FTE* (employees not reported)

\section{Palm Beach County Transportation Agency (Palm Tran)}

Mr. Perry Maull, Executive Director

3201 Electronics Way, West Palm Beach, FL 3340

ach.fl.us/palmtran

Palm Tran is a not-for-profit corporation owned by Palm Beach County that is governed by the seven-member Board of County Commissioners. The corporation provides transit services throughout Palm Beach County. Palm Tran operates founty Com motorbus services and feeder bus services to the Tri-County Commuter Rail system. In addition, Palm Tra provides purchased demand-response services through Spec Tran, the Palm Beach County paratransit division.

\section{General Performance Indicators (FY 2000)}

\subsection{4}

Service'Area Population Density $\quad 2,363.9$
Annul

$\begin{array}{lr}\text { Annual Passenger Trips } & 6,463,416 \\ \text { Annual Revenue Miles } & 6,966 ; 949\end{array}$

Effectiveness Measures (FY 2000) Passenger Trips Per Capita Passenger Trips Per Revenue Mile

Efficiency Measures (FY 2000) Operating Expense Per Capit Operating Expense Per Rev. Mil
Route Miles

Peak Vehicles

Operating Expense

Operating Revenue

Rev. Miles Between Safety Incidents Rev. Miles Between Failures*

\$34.21 Operating Revenue Per Operating Expense

Operating Revenue Per Operating Expense

Average Fare
$1,324.4$

$\$ 27,091,165$

$\$ 5,066,360$

409,82

$18.70 \%$

15,618
$\$ 0.70$
Pasco County Public Transportation (PCPT)

Mr. Michael Carroll, Transportation Manager

(727) Galen Wilson Boulevard, Port Richey, FL 346

Pasco County Public Transportation is a division of Pasco County Government. The division is governed by the five-member Pasco County Commission. Fixed-route motorbus service is provided in the urbanized area of west Pasco County and the municipalities in east Pasco County. Demand-response (advance reservation) service is available throughout Pasco County.

General Performance Indicators (FY 2000)

Service Area Population

Service Area Population Density

Annual Passenger Trips

326,494

429.3

136,142

Route Mles

Peak Vehicles

Operating Expense
Operating Revenue

1

$1,036,28$

Effectiveness Measures (FY 2000)

Passenger Trips Per Capita

0.42 Rev. Miles Between Safety Incidénts

Efficiency Measures (FY 2000)

Operating Expense Per Capit

Operating Expense Per Trip

Operating Expense Per Rev. Mile

Rev. Miles Between Failures

150,011

14,06

Operating Revenue Per Operating Expense Passenger Trips Per Employee FTE

Average Fare

\section{Pinellas Suncoast Transit Authority (PSTA) \\ Mr. Roger Sweeney, Executive Director \\ (727) 530-9921 uww.psta.net}

Pinellas Suncoast Transit Authority is an independent authority that is governed by a Board of Directors comprised of elected

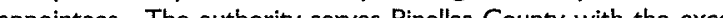
Shores, Treasure Island, and St. Pete Beach. PSTA provides fixed-route motorbus service and demand-response service.

General Performance Indicators (FY 2000) Service Area Population

Service Area Population Density

Annual Passenger Trips

833,504

$9,980.4$

Route Miles

Annual Revenue Miles

$6,776,51$

Operating Expense

Operating Revenue

Effectiveness Measures (FY 2000)

assenger Trips Per Capita

Passenger Trips Per Revenue Mile

11.23
1.38

Rev. Miles Between Safety Incidents

2,828

Efficiency Measures (FY 2000)

Operating Expense Per Capit

Operating Expense Per Capta

Operating Expense Per Rev. Mile
$\$ 31.62 \quad$ Operating Revenuje Per Operating Expense

$\$ 3.89$ Average Fare
$1,703.0$

$\$ 26,355,89$
$\$ 9,800,15$

80,673

$37.18 \%$

$\$ 0.8$

*excludes purchased transportation 


\section{Regional Transit System (Gainesville RTS)}

Mr. Jeff Logan, Executive Directo

(352) 334-2609 www.go-rts.com

Regional Transit System is a department of the City of Gainesville. RTS is governed by an elected five-member Commission. The Commission receives input from an advisory board comprised of citizens from the community. RTS serves the City of throughout the urbanized area up to three-quarters of a mile beyond its fixed-route network.

Service Area Population Density

Annual Passenger Trips

Annual Revenue Miles

Effectiveness Measures (FY 2000)

Passenger Trips Per Capita
Passenger Trips Per Revenue Mile

Efficiency Measures (FY 2000) Operating Expense Per Capit

Operating Expense

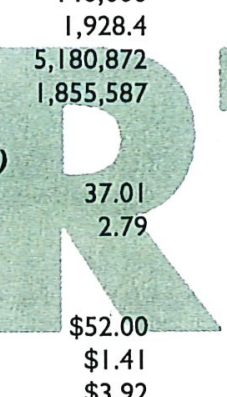

Route Miles

\section{Vehicles}

Operating Expense

58

$\$ 7,279,463$

$\$ 720,830$

Rev. Miles Between Safety Incidents

265,084

Mev Miles Between Failures

$9.90 \%$

Operating Revenue Per Operating Expense

Passenger Trips Per Employee FTE

Average Fare

\section{Sarasota County Area Transit (SCAT)}

Mr. Jay A. Goodwill, P.E., General Manager

(94I) 316-1234 muw.co.sarasota.fl.us/public_works_scat/scat.asp

Sarasota County Transportation Authority, informally known as Sarasota County Area Transit, is a member of the Sarasota County Government. The authority is governed by the five-member Board of County Commissioners. SCAT serves the
urbanized portion of Sarasota County (including the cities of Longboat Key, Sarasota, Venice, Englewood, and North Port) urbanized portion of Sarasota County (including the cities of Lon
via fixed-route motorbus service and demand-response service.

\section{General Performance Indicators (FY 2000)} Service Area Population 278,800 Service Area Population 1,751:3 Annual Passenger Trips

Effectiveness Measures (FY 2000) Passenger Trips Per Capit Passenger Trips Per Revenue Mile Efficiency Measures (FY 2000) Operating Expense Per Capit Operating Expense Per Rev. Mile

Route Miles

$\begin{array}{ll}1,620,586 & \text { Operating Expense } \\ 1,635,128 & \text { Operating Revenue }\end{array}$

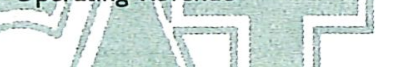

Rev. Miles Between Safety Inciden

Rev. Miles Between Failure

Operating Revenue Per Operating Expense

Passenger Trips Per Employee FTE

Average Fare
Space Coast Area Transit (SCAT)

Mr. James P. Liesenfelt, Transit Director

(321) 635-7815 umpridescatcom

Space Coast Area Transit is a department of Brevard County. The department is governed by the five-member Brevard County Commission. Space Coast provides transit service throughout Brevard County via fixed-route motorbus services, demand-response services, and vanpool services.

General Performance Indicators (FY 2000)

Route Miles

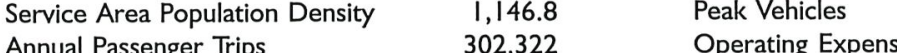

$\begin{array}{lll}\text { Annual Passenger Trips } & 302,322 & \text { Operating Expense } \\ \text { Annual Revenue Miles } & 627,811 & \text { Operating Revenue }\end{array}$

$\$ 1,697,905$

$\$ 389,922$

Effectiveness Measures (FY 2000)

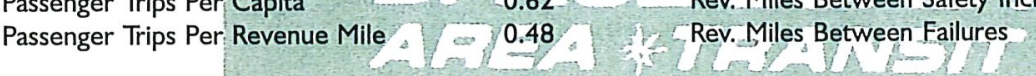

Efficiency Measures (FY 2000)

Operating Expense Per Trip

$\$ 3.47$

Operating Revenúe Per Operating Expense

$22.96 \%$

Operating Expense Per Rev. M

$\$ 2.70$

Average Fare

\section{Tallahassee Transit (TALTRAN) \\ Mr. John L. Carter, Director}

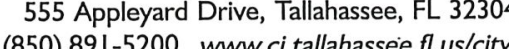

TALTRAN is a department of the City of Tallahassee government. It is governed by a Board of Directors that is comprised of four elected commissioners and one elected mayor. TALTRAN serves the City of Tallahassee by providing fixed-route motorbus service and demand-response service to its community. Evening, weekend, and holiday demand-response services are contracted with Intelitran.

\section{General Performance Indicators (FY 2000)}

Service Area Population

$\begin{aligned} 147,490 & \text { Route Miles } \\ 1,505.0 & \text { Peak Vehicles } \\ 3,922,150 & \text { Operating Expense } \\ 1,678,460 & \text { Operating Revenue }\end{aligned}$

$\$ 4,968,718$

$\$ 1,054,919$

Annual Revenue Miles

Operating Revenu

Effectiveness Measures (FY 2000) Passenger Trips Per Capi

ger Trips Per Revenue Mile

$21.23 \%$

Efficiency Measures (FY 2000)

Operating Expense Per Capi

Operating Expense Per Rev. Mile
26.59 Rev. Miles Between Safety Incidents

2.34 Rev. Miles Between Failures

$\mathrm{HL} \quad \mathrm{DH}$

\$52.20 Operating Revenue Per Operating Expense

\$1.96 Passenger Trips Per Employee FTE

$\$ 4.59 \quad$ Average Fare
201.0

$\$ 7,698,262$ $\$ 2,785,420$ 
Tri-County Commuter Rail Authority (Tri-Rail)

Mr. Joseph Giuletti, Executive Director

800 NW 33rd Street, Suite 100, Pompano Beach, FL 33064

(800) TRI-RAIL www.tri-rail.com

The Tri-County Commuter Rail Authority is an independent authority established by the Florida Legislature. The authority is governed by a nine-member appointed Board of Directors. Tri-Rail operates and manages the commuter rail system for Broward, Dade, and Palm Beach Counties.

\section{General Performance Indicators (FY 2000)}

Service Area Population $\quad 4,659,18$

$\begin{array}{lr}\text { Service Area Population Density } & 3,477.0 \\ \text { Annual Passenger Trips } & 2,232,497\end{array}$

Annual Passenger Trips

$1,819,317$

Route Miles

Peak Vehicles

Operating Expense

Annual Revenue Miles

Operating Revenue

$\$ 20,572,46$

$\$ 6,149,262$

Effectiveness Measures (FY 2000)

Passenger Trips Per Capita

$0.48^{\circ} \quad$ Rev. Miles Between Sáfety Incidents

139,947

Passenger Trips Per Revenue M

Efficiency Measures (FY 2000)

Operating Expense Per Capit

1.23

ev. Miles Between Fallures /

Operating Expense Per Rev. Mile

Operating Revenue Per Operating Expense
Passenger Trips

$\$ 2.30$$$
\text { Average Fare }
$$

\section{County of Volusia dba VOTRAN \\ r. Kenneth R. Fischer, General Manager \\ 50 Big Tree Road, South Daytona, FL 32$$
\text { (386) } 756-7496 \text { www.votran.org }
$$

VOTRAN is a part of Volusia County's General Fund. It is governed by a Board of Directors that is comprised of seven County Council members. VOTRAN provides public transportation services throughout Volusia County via fixed-route motorbus services and paratransit services.

\section{General Performance Indicators (FY 2000)} Annual Passenger Trips Density $\quad 348.3$ Annual Revenue Miles $4,046,072$ Route Miles Peak Vehicle

Operating Expense
Operating Revenue

Effectiveness Measures (FY 2000)

(n) $2,531,865$

$\$ 7,317,569$

$\$ 1,704,753$

Passenger Trips Per Revenue Mil

Rev. Miles Between Safety Incidents Rev. Miles Between Failures

194,759

Efficiency Measures (FY 2000) Operating Expense Per Capit

Operating Expense Per Rev. Mil

Winter Haven Area Transit (WHAT)

290 Golfview Avenue, Drawer HSO9, P.O. Box 9005, Bartow, FL 3383

(863) 534-5301 www.Polk-County.net

Winter Haven Area Transit is a new transit system that began operating in 1999. The Citrus Connection system of Lakeland has been contracted to provide the fixed-route motorbus system in the City of Winter Haven. The new system is governed by the WHAT Policy Board.

\section{Service Area Populatio \\ Service Area Population Density \\ Annual Passenger Trips \\ Annual Revenue Miles}

Effectiveness Measures (FY 2000) assenger Trips Per Capit

Renue Mile

\section{Efficiency Measures (FY 2000)}

Operating Expen

Operating Expense Per Trip

Operating Expense Per Rev. Mile

*excludes purchased transportation
Route Miles

3. Peak Vehicles

83,929 Operating Revenue

127.8

$\$ 520,811$

$\$ 41,16$

1.13 Rev. Miles Between Safety Incidents (zero incidents reported) 0.53 Rev. Miles Between Failures

22,991

10.0 Operating Revenue Per Operating Expense ps Per Employee FTE* $\$ 2.83$ Average Fare 

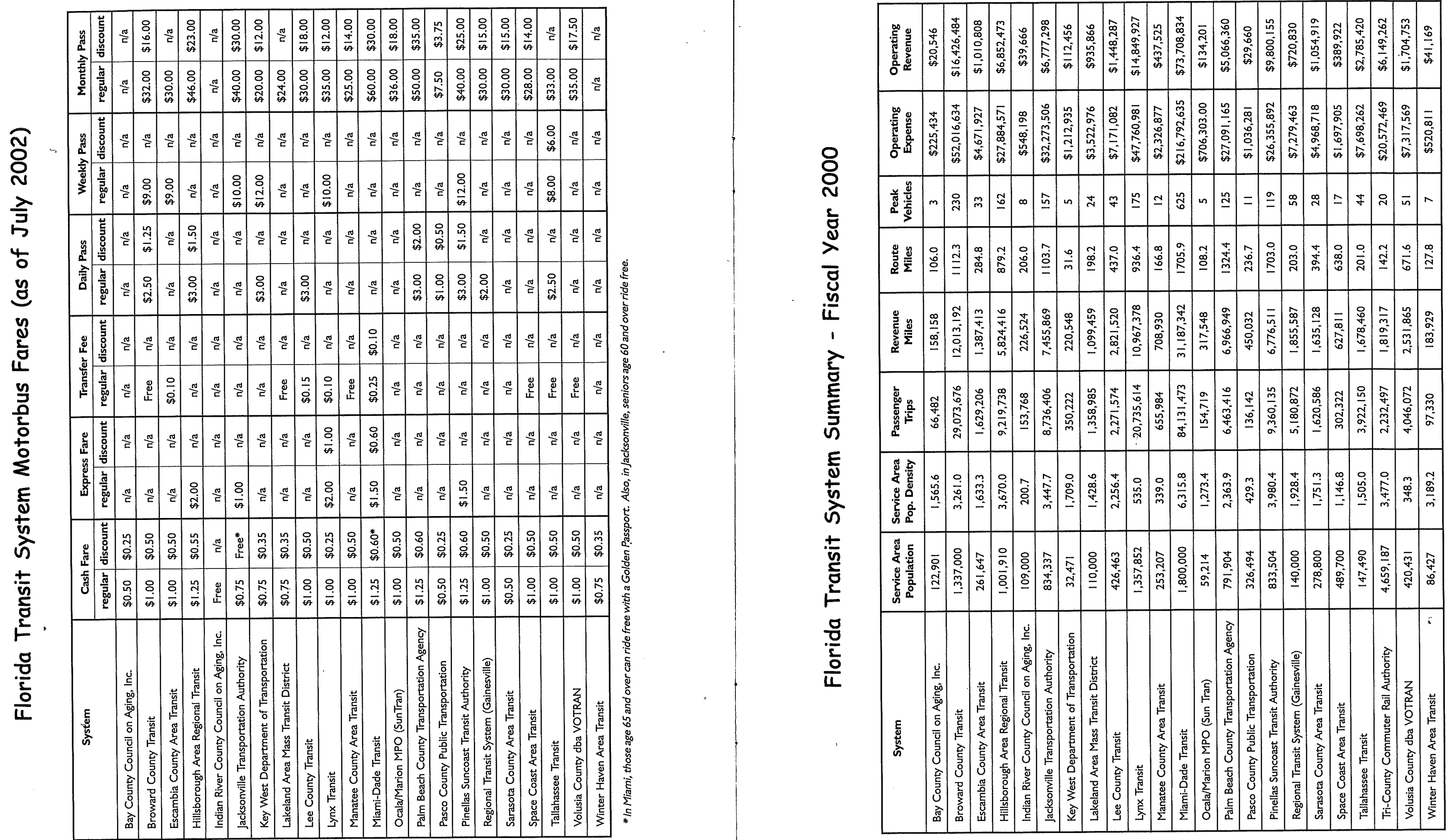

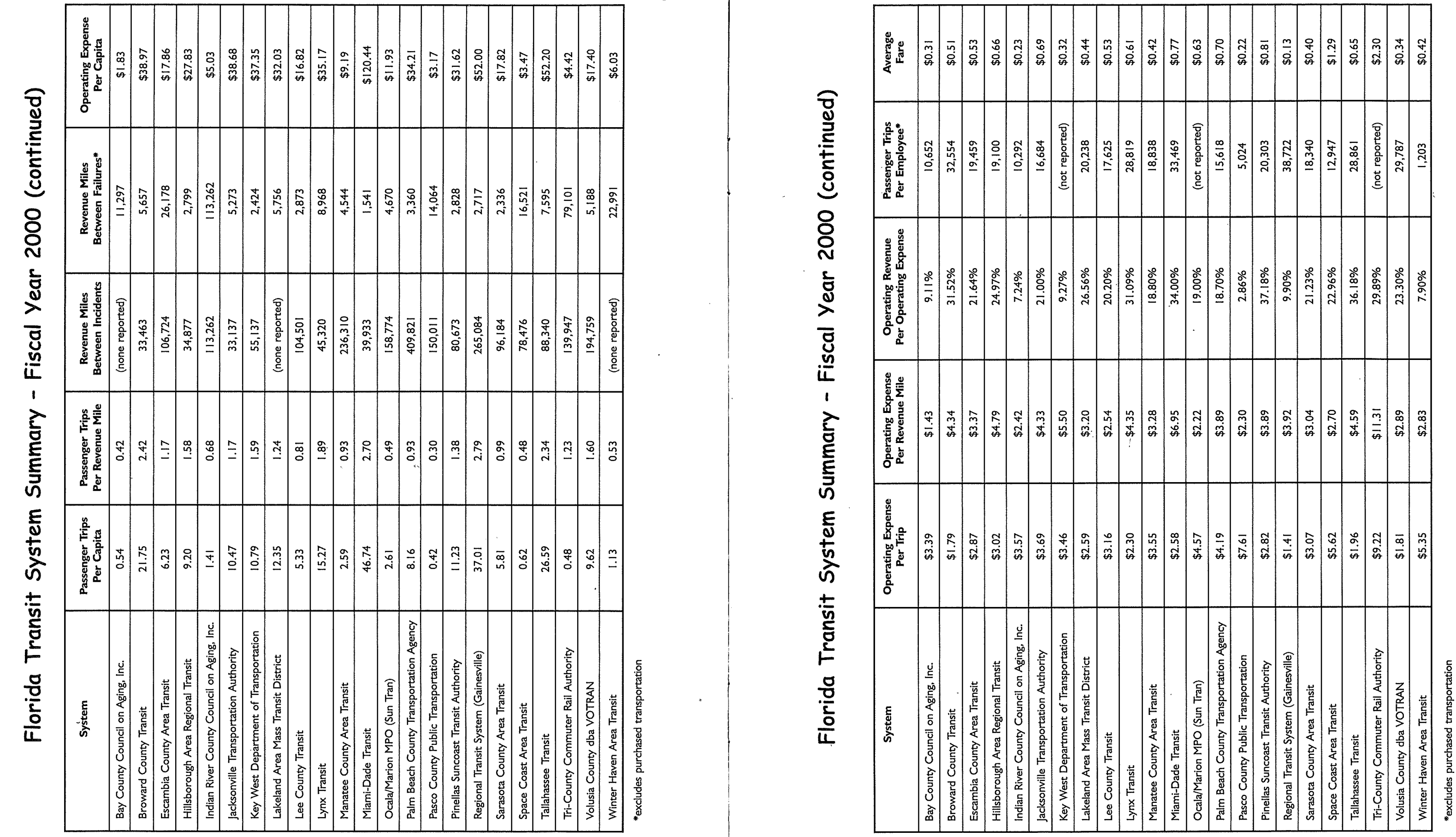
Florida's Transit Systems-FY 2000 Statewide Totals

FDOT Public Transit Office

(850) 414-4500 http://www11.myflorida.com

In fiscal year 2000, 26 transit systems in Florida received State Block Grant funds. Twenty-two systems provide fixed-route motorbus; 20 or these also provide demand-response service. Three provide only demand-response service. The remainin system, Tri-Rail, operates commuter rall service in South Horida. Also, Miami-Dade Transit operates heavy rail (Metroral)

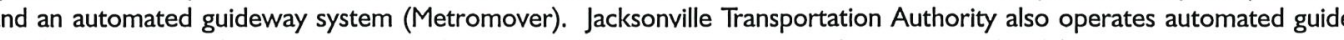
way (Skyway Express). Please see pages 6 and 7 of this Handbook for more information on Florida's transit systems.

\section{General Performance Indicators (FY 2000)}

Service Area Population

$11,220,752$
$1,260.1$

$191,899,369$

Route Miles

$12,918.2$

Peak Vehicles

Operating Revenue

$\$ 501,742,594$

$\$ 150,496,82$

Effectiveness Measures (FY 2000) Passenger Trips Per Capita Passenger Trips Per Revenue Mile

Efficiency Measures (FY 2000)

Operating Expense Per Capita

Operating Expense Per Trip

Operating Expense Per Rev. Mile

*excludes purchased transportation data
There are numerous factors that affect the performance of individual transit systems. These factors, illustrated below, can be difficult to quantify, but must be considered since they comprise the most unique features of each transit agency and the community it serves.
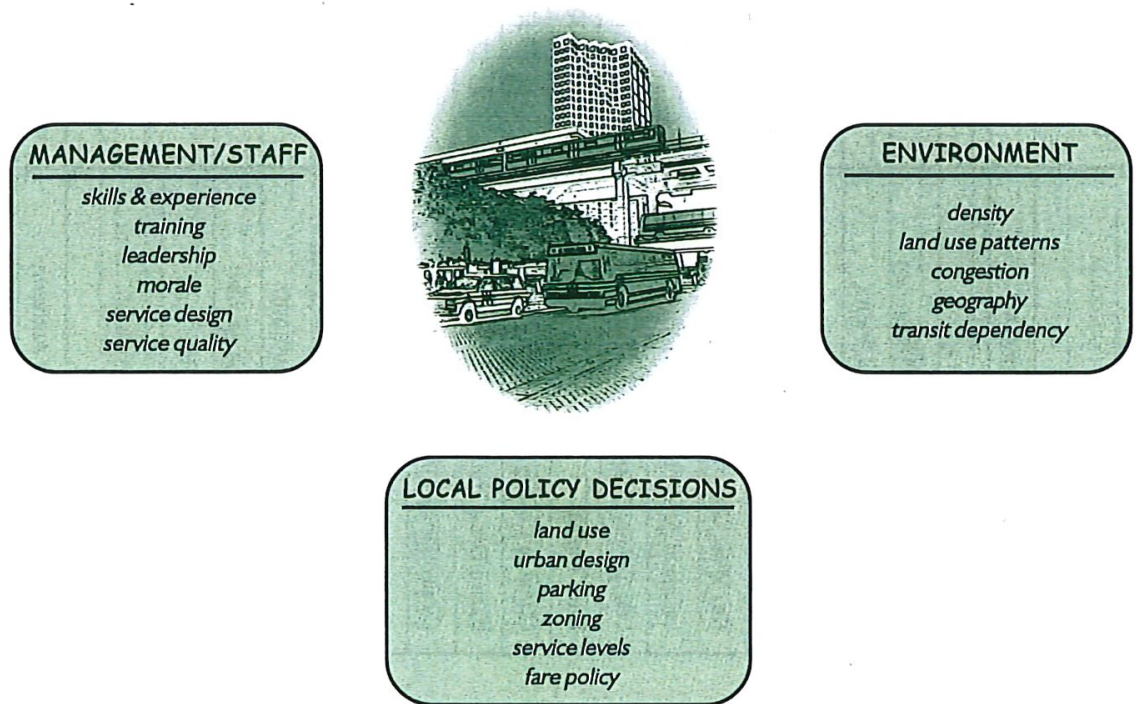

\section{Glossary of Terms}

Automated Guideway - A fixed-guideway transit system that operates with automated (driverless) individual vehicles or multi-car trains. Service may be on a fixed schedule or in response to a passenger-activated call button.

Commuter Rail - Short-haul rail passenger service operating in metropolitan and suburban areas, whether within or across the geographical boundaries of a state, usually characterized by reduced fare, multiple ride, and commutation tickets and by morning and evening peak period operations.

Demand-Response Service - Any system of transporting individuals, including the provision of designated transportation service by public or private entities, which is not a fixed-route sys tem. This type of service is also known as "paratransit."

Fixed Guideway - Any public transportation facility that utilizes and occupies a separate rightof-way or rails for the exclusive use of public transportation service including, but not limited to, fixed rail, automated guideway transit, and exclusive facilities for buses and other highoccupancy vehicles.

Heavy Rail - Transit service using rail cars with motive capability, driven by electric power usually drawn from a third rail, configured for passenger traffic, and usually operated on exclusive rights-of-way.

Motorbus - Mode of service involving passenger revenue vehicles operating on fixed routes and schedules on roadways.

Park-and-Ride - Parking garages and/or pavement used for parking passengers' automobiles, either free or for a fee, while they use transit system facilities. The garages/lots are generally used as collector sites for rail or bus service. They may also serve as collector sites for vanpools or carpools, and may sometimes serve as transit centers.

Purchased Transportation - Transportation service provided to a public agency from a public or private transportation provider based on a written contract. It does not include franchising licensing operations, management services, or private conventional bus service.

GENERALPERFORMANCEINDICATORS

Annual Passenger Trips - Annual number of passenger boardings on the transit vehicles. A trip is counted each time a passenger boards a transit vehicle. Thus, if a passenger has to transfer between buses to reach a destination, he/she is counted as making two passenger trips.

Annual Revenue Hours - Total hours of operation by revenue service vehicles in active revenue service.

Annual Revenue Miles - Number of annual miles of vehicle operation while in active service (available to pick up revenue passengers). This number is smaller than vehicle miles because of 
the exclusion of deadhead miles such as vehicle miles from the garage to the start of service, vehicle miles from the end of service to the garage, driver training, and other miscellaneous miles that are not considered to be in direct revenue service.

Annual Vehicle Hours - Total hours of operation by revenue service vehicles including hours consumed in passenger service and deadhead travel.

Annual Vehicle Miles - Total distance traveled annually by revenue service vehicles, including both revenue miles and deadhead miles.

Number of Safety Incidents - Total number of unforeseen occurrences resulting in casualty (injury/fatality), collision, or property damage in excess of $\$ 1,000$. For an incident to be reportable, it must involve a transit vehicle or occur on transit property.

Operating Revenue - All revenues generated through the operation of the transit agency. Includes passenger fares, special transit fares, school bus service revenues, freight tariffs, charter service revenues, auxiliary transportation revenues, subsidy from other sectors of operations, and non-transportation revenues.

Passenger Fare Revenue - Revenue generated annually from carrying passengers in regularly scheduled service.

Peak Vehicles - This refers to the number of vehicles operated in maximum (peak) service. It represents the number of revenue vehicles operated to meet the annual maximum service requirement, i.e., the revenue vehicle count during the peak hours of the peak days/weeks of the peak season (typically the rush period). Vehicles operated in maximum service exclude atypical days or one-time special events.

Route Miles - Number of directional route miles as reported in NTD data; defined as the mileage that service operates in each direction over routes traveled by public transportation vehicles in revenue service.

Service Area Population - This indicator provides a suitable approximation of overall market size for comparison of relative spending and service levels among communities in the absence of actual service area population. In 1992, the Federal Transit Administration (FTA) began requiring transit systems to provide service area population in their Section I5/NTD reports.

Total Employee FTEs - Total number of payroll employees of the transit agency in terms of fulltime equivalents (FTEs). All employees classified as capital are not included in this Handbook.

Total Operating Expense - Reported total spending on operations, including administration, maintenance, and operation of service vehicles.

Vehicle System Failures - Total number of failures that occur during revenue service, deadheading, and layovers. A failure is classified as the breakdown of either a major or minor element of the revenue vehicle's mechanical system. Failures are tabulated regardless of whether they result in a vehicle completing or not completing its trip.

\section{EFFECTIVENESSMEASURES}

Passenger Trips Per Capita - Average number of transit boardings per person per year. This number is larger in areas where public transportation is emphasized and in areas where there are more transit dependent persons, and is a measure of the extent to which the public utilizes transit in a given service area.

Passenger Trips Per Revenue Mile - The ratio of passenger trips to revenue miles of service; a key indicator of service effectiveness that is influenced by the levels of demand and the supply of service provided.

Revenue Miles Between Failures - Number of revenue miles divided by the number of revenue vehicle system failures; an indicator of the average frequency of delays because of a problem with the equipment.

Revenue Miles Between Safety Incidents - Number of revenue miles divided by the number of incidents; reports the average interval, in miles, between incidents.

\section{EFFICIENCYMEASURES}

Average Fare - Passenger fare revenues divided by the total number of passenger trips.

Farebox Recovery Ratio - Ratio of passenger fares to total operating expenses; an indicator of the share of total operating costs that is covered by the passengers' fares.

Maintenance Expense Per Revenue Mile - Maintenance costs divided by the revenue miles.

Operating Expense Per Capita - Annual operating budget divided by the service area population; a measure of the resource commitment to transit by the community.

Operating Expense Per Passenger Trip - Operating expenditures divided by the total annual ridership; a measure of the efficiency of transporting riders; one of the key indicators of comparative performance of transit properties since it reflects both the efficiency with which service is delivered and the market demands for the service.

Operating Expense Per Revenue Mile - Operating expense divided by the number of revenue miles of service; a measure of the efficiency with which service is delivered and is another key comparative indicator.

Operating Revenue Per Operating Expense - Ratio of revenue generated through operation of the transit agency with respect to total operating expense.

Passenger Trips Per Employee FTE - Ratio of total passenger trips to system total full-time equivalents. Another measure of overall labor productivity.

Revenue Hours Per Employee FTE - Ratio of total revenue hours of service to system total fulltime equivalents; reflects overall labor productivity. 
Center for Urban Transportation Research College of Engineering, University of South Florida

$$
\begin{aligned}
& \text { (1) Engineering, University of South Flo } \\
& 4202 \text { East Fowler Avenue, CUT I00 }
\end{aligned}
$$

Tampa, FL 33620-5375
(813) 974-3!20, Fax (813) 974-5 I68, SunCom 574-3120 website: http://www. cutr.usf.edu 